ARTICLE

\title{
A steep-slope transistor based on abrupt electronic phase transition
}

Nikhil Shukla1, Arun V. Thathachary¹, Ashish Agrawal1', Hanjong Paik², Ahmedullah Aziz' ${ }^{1}$ Darrell G. Schlom ${ }^{2,3}$, Sumeet Kumar Gupta1, Roman Engel-Herbert ${ }^{4} \&$ Suman Datta ${ }^{1}$

Collective interactions in functional materials can enable novel macroscopic properties like insulator-to-metal transitions. While implementing such materials into field-effect-transistor technology can potentially augment current state-of-the-art devices by providing unique routes to overcome their conventional limits, attempts to harness the insulator-to-metal transition for high-performance transistors have experienced little success. Here, we demonstrate a pathway for harnessing the abrupt resistivity transformation across the insulator-to-metal transition in vanadium dioxide $\left(\mathrm{VO}_{2}\right)$, to design a hybrid-phase-transition field-effect transistor that exhibits gate controlled steep ('sub- $\mathrm{K} T / \mathrm{q}^{\prime}$ ) and reversible switching at room temperature. The transistor design, wherein $\mathrm{VO}_{2}$ is implemented in series with the field-effect transistor's source rather than into the channel, exploits negative differential resistance induced across the $\mathrm{VO}_{2}$ to create an internal amplifier that facilitates enhanced performance over a conventional field-effect transistor. Our approach enables low-voltage complementary n-type and p-type transistor operation as demonstrated here, and is applicable to other insulator-to-metal transition materials, offering tantalizing possibilities for energy-efficient logic and memory applications.

\footnotetext{
${ }^{1}$ Department of Electrical Engineering, Pennsylvania State University, University Park, Pennsylvania 16802, USA. ${ }^{2}$ Department of Materials Science and Engineering, Cornell University, Ithaca, New York 14853, USA. ${ }^{3}$ Kavli Institute at Cornell for Nanoscale Science, Ithaca, New York 14853 , USA. ${ }^{4}$ Department of Materials Science and Engineering, Pennsylvania State University, University Park, Pennsylvania 16802, USA. Correspondence and requests for materials should be addressed to S.D. (email: sdatta@engr.psu.edu).
} 
M etal-oxide-semiconductor field-effect transistors (MOSFETs) have been the workhorse of digital computation. In a conventional MOSFET (Fig. 1a), a change in the drain-to-source current $\left(I_{\mathrm{DS}}\right)$ can be induced by the application of a transverse electric field across the gate dielectric by means of the third gate terminal. This field lowers the potential energy barrier separating the source and the channel, exponentially increasing the number of carriers traversing the channel. At room temperature, a minimum change of $60 \mathrm{mV}$ in the gate bias $\left(V_{\mathrm{GS}}\right)$ is required to effectuate a decade change in $I_{\mathrm{DS}}$, setting up the so-called ' $60 \mathrm{mV}$ per decade' limit, also known as the 'Boltzmann limit' (Fig. 1a). Stemming from the statistical distribution of free and independent carriers in conventional semiconductors and determined by the thermal voltage $k T / q$ ( $k$ : Boltzmann constant; $T$ : temperature; $q$ : electron charge), this fundamental limit restricts transistor performance, particularly at low-operating voltages ${ }^{1-4}$, and has motivated the exploration of FETs that harness collective carrier responses ${ }^{5-13}$. Such collective behaviour-wherein a small external perturbation can trigger an aggregated change in the ground state of the system-can produce internal amplification; and provide a pathway to overcome the Boltzmann limit to enable FET's with sub- $k T / q$ $(k T / \eta q ; \eta>1)$ switching slope and superior performance at low voltages. Particularly, in insulator-to-metal transition (IMT) materials ${ }^{14}$ that exhibit strong correlation, like $\mathrm{VO}_{2}$ (refs 15-18), the collective response to external perturbation (temperature ${ }^{19,20}$, pressure $^{21,22}$ and electrical stimulus ${ }^{23-28}$ ) can be the 'melting' of carriers, marking an electronic phase transformation where the electrons localized at atomic sites change to an itinerant state (Fig. 1b). This phase transformation amplifies the free-carrier concentration ${ }^{29}$; and in the case of $\mathrm{VO}_{2}$, manifests itself as a sharp change in resistivity up to five orders in magnitude ${ }^{30}$ at $\sim 340 \mathrm{~K}$. However, attempts to realize IMT-based three-terminal transistor devices with a solid-state gate dielectric to induce the phase transition directly in the channel material have experienced only limited success ${ }^{23,31-33}$. Further, the alternate approach of using an ionic liquid as the gate dielectric, which is the focus of current research ${ }^{34-39}$, is typically slow ${ }^{40-42}$ and susceptible to electrochemical effects ${ }^{43}$. These constraints have restricted the utilization of this collective phenomenon in FETs for advanced high-performance electronic applications.

Here, we explore a novel transistor architecture that harnesses the abrupt free-carrier amplification across the phase transition in $\mathrm{VO}_{2}$ using a conventional MOSFET. By electrically coupling the $\mathrm{VO}_{2}$ in series with the source of a conventional MOSFET (Fig. 1b), we design a hybrid-phase-transition-FET (hyper-FET) wherein, for a given drain-to-source voltage $\left(V_{\mathrm{DS}}\right)$, the gate bias $V_{\mathrm{GS}}$ modifies the current $I_{\mathrm{DS}}$ flowing through the MOSFET channel and the $\mathrm{VO}_{2}$ in series, triggering an abrupt phase transformation in $\mathrm{VO}_{2}$. The proposed hyper-FET not only exhibits steep-slope characteristics but also circumvents the need for a direct field-induced phase transition in $\mathrm{VO}_{2}$ with a solidstate gate dielectric. Further, the abrupt resistivity switching of $\mathrm{VO}_{2}$ in the hyper-FET configuration, which is the origin of the steep-slope characteristics, induces a negative differential resistance (NDR) across $\mathrm{VO}_{2}$ that results in internal voltage amplification which consequently enhances the hyper-FET's performance beyond that of a conventional MOSFET.

\section{Results}

Experimental demonstration and operation principle. Figure 2a illustrates the schematic of an experimental hyper-FET consisting of a two-terminal $\mathrm{VO}_{2}$ device in series with the channel of a conventional Si n-MOSFET (individual device characteristics are shown in Supplementary Fig. 1 and discussed in Supplementary Note 1). All measurements in this work are performed at room
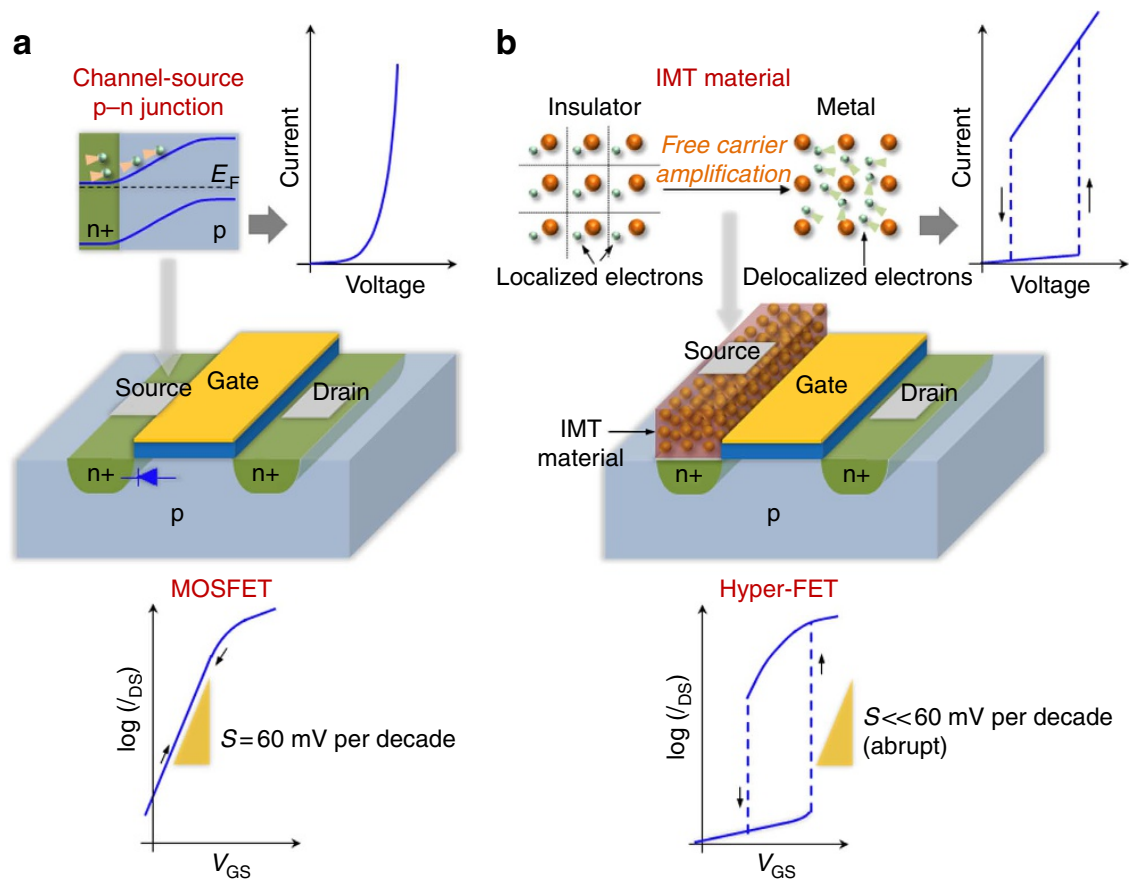

Figure 1 | Schematic device design of hyper-FET and working principle. (a) Conventional MOSFET and its transfer characteristics (channel current $I_{D S}$ versus gate bias $V_{G S}$ ) for a fixed drain-to-source voltage $V_{D S}$. The channel-source $p-n$ junction, modulated by the gate terminal, controls the injection of carriers into the channel limiting the switching slope $(S)$ of the MOSFET to $60 \mathrm{mV}$ per decade (Boltzmann limit). (b) Proposed hyper-FET in which an insulator-to-metal transition (IMT) material that shows electrically induced abrupt resistivity switching is electrically integrated in series with the source of a conventional MOSFET. For a given $V_{D S}$, the gate-terminal voltage $V_{G S}$ modifies the current flowing through the MOSFET and the IMT material in series, triggering an abrupt phase transition. The associated delocalization of localized carriers (free-carrier amplification) across the IMT results in an abrupt decrease in the resistance of the source, enhancing the switching slope characteristics beyond the intrinsic limits of a conventional $p-n$ junction. 

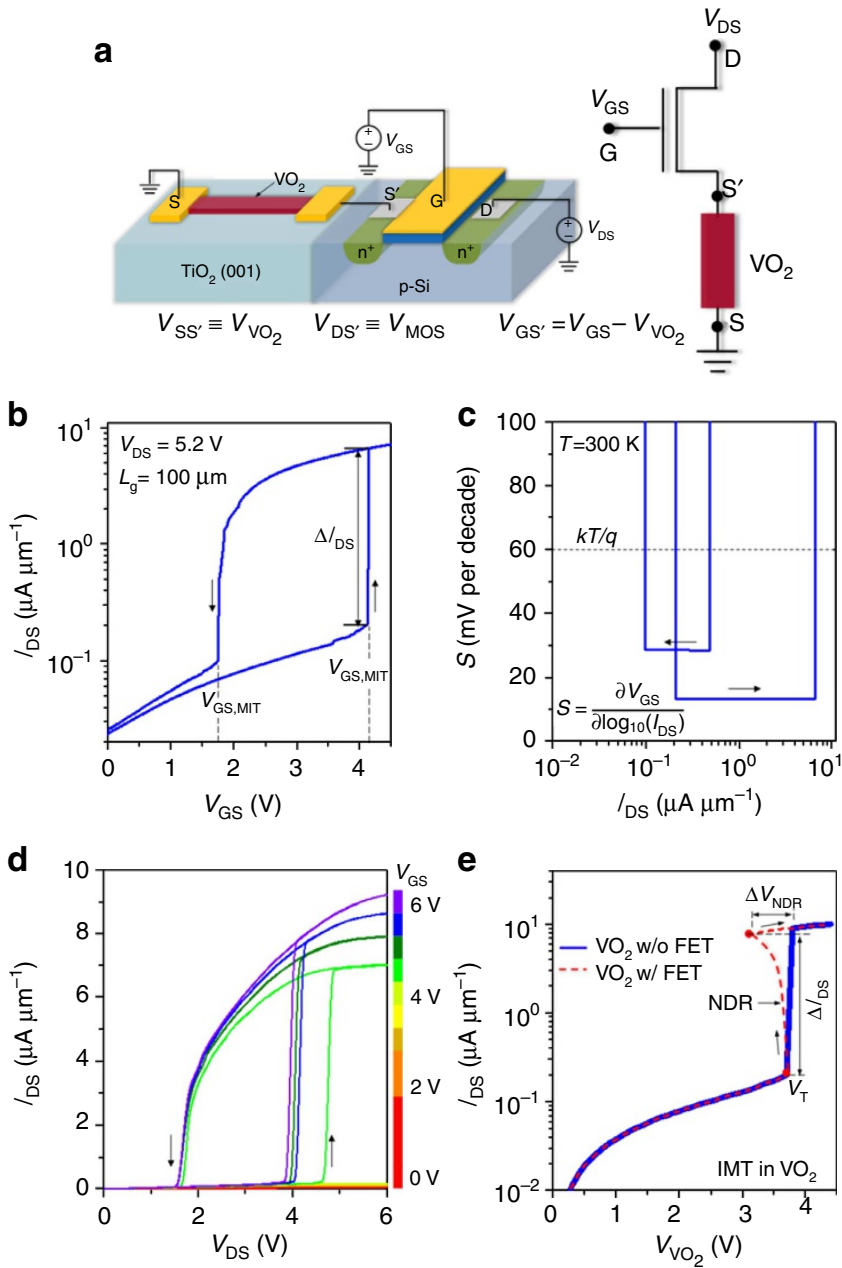

Figure 2 | Experimental demonstration of a $\mathbf{V O}_{2}$-based hyper-FET.

(a) Schematic of a hyper-FET consisting of a two-terminal $\mathrm{VO}_{2}$ device $\left(L_{\mathrm{VO}_{2}}=4 \mu \mathrm{m} ; W_{\mathrm{VO}_{2}}=2 \mu \mathrm{m}\right)$ in series with the channel of a conventional $\mathrm{Si}$ $\mathrm{n}$-MOSFET $\left(L_{\mathrm{g}}=100 \mu \mathrm{m} ; \mathrm{W}=100 \mu \mathrm{m}\right) . V_{\mathrm{VO}_{2}}$ is the voltage across the $\mathrm{VO}_{2}$ device and $V_{G S^{\prime}}$ is the effective gate-to-source voltage across the MOSFET. (b) $I_{\mathrm{DS}}-V_{\mathrm{GS}}$ transfer characteristics of the hyper-FET exhibiting abrupt and reversible modulation of the channel current $I_{D S}$ as a function of the gate-source voltage $V_{\mathrm{GS}}$. The abrupt turn-ON and turn-OFF of the hyper-FET corresponds to the IMT and MIT in $\mathrm{VO}_{2}$, respectively.

(c) Switching slope $(S)$ as a function of $I_{D S}$ revealing the steep-slope characteristics ( $S<60 \mathrm{mV}$ per decade) of the hyper-FET during the forward and reverse gate bias sweep. (d) Output characteristics $\left(I_{D S}-V_{D S}\right)$ of the hyper-FET with excellent current saturation. (e) Current versus voltage characteristics of the $\mathrm{VO}_{2}$ device with (red) and without (blue) the MOSFET in series, illustrating the electrically triggered abrupt IMT. The channel resistance of the MOSFET acts as a series resistor, modifying the current-voltage dynamics through a feedback and inducing a negative differential resistance NDR (red) across the phase transition in $\mathrm{VO}_{2}$. The NDR reduces the voltage across the $\mathrm{VO}_{2}$ by $\Delta V_{\text {NDR }}$. The current has been normalized to the width of the Si n-MOSFET to show that the abrupt IMT in $\mathrm{VO}_{2}$ triggers the abrupt turn-ON of the hyper-FET shown in $\mathbf{b}$.

temperature $(T=300 \mathrm{~K})$. The modulation in the transfer characteristics $\left(I_{\mathrm{DS}}-V_{\mathrm{GS}}\right)$ of the hyper-FET is shown in Fig. $2 \mathrm{~b}$. Initially, at $V_{\mathrm{GS}}=0 \mathrm{~V}$ (MOSFET in OFF-state), $\mathrm{VO}_{2}$ is in the high-resistivity insulating state. In this series combination, $V_{\mathrm{DS}}$ is divided between the MOSFET channel and the insulating $\mathrm{VO}_{2}$ in proportion to their respective resistances; and the current $I_{\mathrm{DS}}$ through the channel and $\mathrm{VO}_{2}$ is insufficient to induce an IMT. As $V_{\mathrm{GS}}$ increases, the MOSFET-channel resistance decreases until
$I_{\mathrm{DS}}$ reaches a critical current threshold, $I_{\mathrm{IMT}}$. This triggers an abrupt IMT with the $\mathrm{VO}_{2}$ transforming into the low-resistivity metallic state that consequently leads to an abrupt increase in $I_{\mathrm{DS}}$ (turn-ON). Similarly, as $V_{\mathrm{GS}}$ reduces, the MOSFET-channel resistance increases until $I_{\mathrm{DS}}$ drops to a critical threshold value, $I_{\text {MIT }}$, and the $\mathrm{VO}_{2}$ transforms back to the high-resistivity insulating state accompanied by an abrupt reduction in $I_{\mathrm{DS}}$ (turn-OFF). The difference between the critical threshold values ( $I_{\mathrm{MIT}}>I_{\mathrm{IMT}}$; corresponding to $V_{\mathrm{GS}, \mathrm{IMT}}, V_{\mathrm{GS}, \mathrm{MIT}}$, respectively) results in hysteresis $\left(=V_{\mathrm{GS}, \mathrm{IMT}}-V_{\mathrm{GS}, \mathrm{MIT}}\right)$ (Fig. 2b).

Analysing the switching slope $S=\frac{\partial V_{\mathrm{GS}}}{\partial \log _{10}\left(I_{\mathrm{DS}}\right)}$, shown in Fig. $2 \mathrm{c}$, it is evident that the abrupt change in current associated with the IMT/MIT in $\mathrm{VO}_{2}$ results in steep-slope $(S<60 \mathrm{mV}$ per decade) characteristics, both during the forward and the reverse $V_{\mathrm{GS}}$ sweep. We emphasize that the current change $\Delta I_{\mathrm{DS}}$ is abrupt and the extracted value of $S$ is limited by the voltage resolution $(1 \mathrm{mV})$. The corresponding output characteristics $\left(I_{\mathrm{DS}}-V_{\mathrm{DS}}\right)$ of the hyper-FET (Fig. 2d) show excellent $I_{\mathrm{DS}}$ saturation behaviour, which is paramount for small signal amplification. This is in contrast to the traditional IMT-based transistor design where the IMT occurs in the channel material ${ }^{23}$. Such a transistor is unlikely to demonstrate current saturation since the design envisages the IMT channel to have a metallic character in the transistor's $\mathrm{ON}$-state which fundamentally cannot sustain a drain side depletion region.

Internal amplification in the hyper-FET. To elucidate the internal amplification, we analyze the current-voltage dynamics across $\mathrm{VO}_{2}$ in the hyper-FET configuration (Fig. 2e). In this series combination, the abrupt IMT results in an NDR across the $\mathrm{VO}_{2}$. Such an NDR is induced because when the $\mathrm{VO}_{2}$ resistance decreases abruptly, it results in (a) an increase in $I_{\mathrm{DS}}\left(\Delta I_{\mathrm{DS}}\right)$ which flows through the $\mathrm{VO}_{2}$ device and the MOSFET channel in series; (b) a reduction in the voltage across the $\mathrm{VO}_{2}$ device $V_{\mathrm{VO}_{2}}$ $\left(-\Delta V_{\mathrm{NDR}}\right)$ (see Supplementary Fig. 2 and Supplementary Note 2 for discussion on the NDR in $\left.\mathrm{VO}_{2}\right)$. The effective gate-to-source voltage across the MOSFET $\left(V_{\mathrm{GS}^{\prime}}\right.$; $\mathrm{S}^{\prime}$ : internal node in Fig. 2a) when $\mathrm{VO}_{2}$ is in the insulating state (hyper-FET OFF-state) is $V_{\mathrm{GS}^{\prime}}=V_{\mathrm{GS}}-V_{\mathrm{VO}_{2}}$. It can be observed that the voltage across the insulating $\mathrm{VO}_{2}$ results in an additional voltage drop $\left(=-V_{\mathrm{VO}_{2}}\right)$ in the effective gate-to-source voltage $V_{\mathrm{GS}}$. Across the IMT in $\mathrm{VO}_{2}$ which induces the $\mathrm{NDR}$, this voltage drop $\left(=-V_{\mathrm{VO}_{2}}\right)$ reduces by $\Delta V_{\mathrm{NDR}}$ (therefore increasing $V_{\mathrm{GS}^{\prime}}$ by $\Delta V_{\mathrm{NDR}}$; Fig. 2a). Thus, the additional voltage drop $\left(=-V_{\mathrm{VO}_{2}}\right)$ in $V_{\mathrm{GS}^{\prime}}$ when $\mathrm{VO}_{2}$ is in the insulating state results in a drastic reduction in the OFFstate current $\left(I_{\mathrm{DS}, \mathrm{OFF}}\right)$ of the hyper-FET in comparison to the stand-alone MOSFET, whereas the reduction in ON-state current $\left(I_{\mathrm{DS}, \mathrm{ON}}\right)$ is much less significant since the voltage drop across the metallic $\mathrm{VO}_{2}$ is small; this results in an overall enhanced current change that is, a higher $I_{\mathrm{DS}, \mathrm{ON}} / I_{\mathrm{DS}, \mathrm{OFF}}$ ratio (see Supplementary Fig. 3 and Supplementary Note 3 for additional details and simulations). We model the MOSFET with $\mathrm{VO}_{2}$ combination as an equivalent common-source transistor circuit (Fig. 2a) where:

$$
\left|\frac{\Delta I_{\mathrm{DS}}}{\Delta V_{\mathrm{GS}^{\prime}}}\right|=\frac{g_{\mathrm{m}}}{1+g_{\mathrm{m}} R_{\mathrm{VO}_{2}}}
$$

Here, $g_{\mathrm{m}}$ is the transconductance of the stand-alone MOSFET. Across the IMT, the $\mathrm{VO}_{2}$ exhibits an NDR $\left(R_{\mathrm{VO}_{2}}=\right.$ $\left.-\Delta V_{\mathrm{NDR}} / \Delta I_{\mathrm{DS}}\right)$, and therefore equation (1) evolves to:

$$
\left|\frac{\Delta I_{\mathrm{DS}}}{\Delta V_{\mathrm{GS}}}\right|_{\mathrm{IMT}, \text { hyper - FET }}=\frac{g_{\mathrm{m}}}{1-g_{\mathrm{m}}\left(\frac{\Delta V_{\mathrm{NDR}}}{\Delta \mathrm{IDS}_{\mathrm{DS}}}\right)_{\mathrm{IMT}}}=\beta g_{\mathrm{m}}
$$

where $\beta=\frac{1}{1-g_{\mathrm{m}} \cdot\left(\frac{\Delta V_{\mathrm{NDR}}}{\Delta \mathrm{IDS}_{\mathrm{DS}}}\right)_{\mathrm{IMT}}}>1$ 

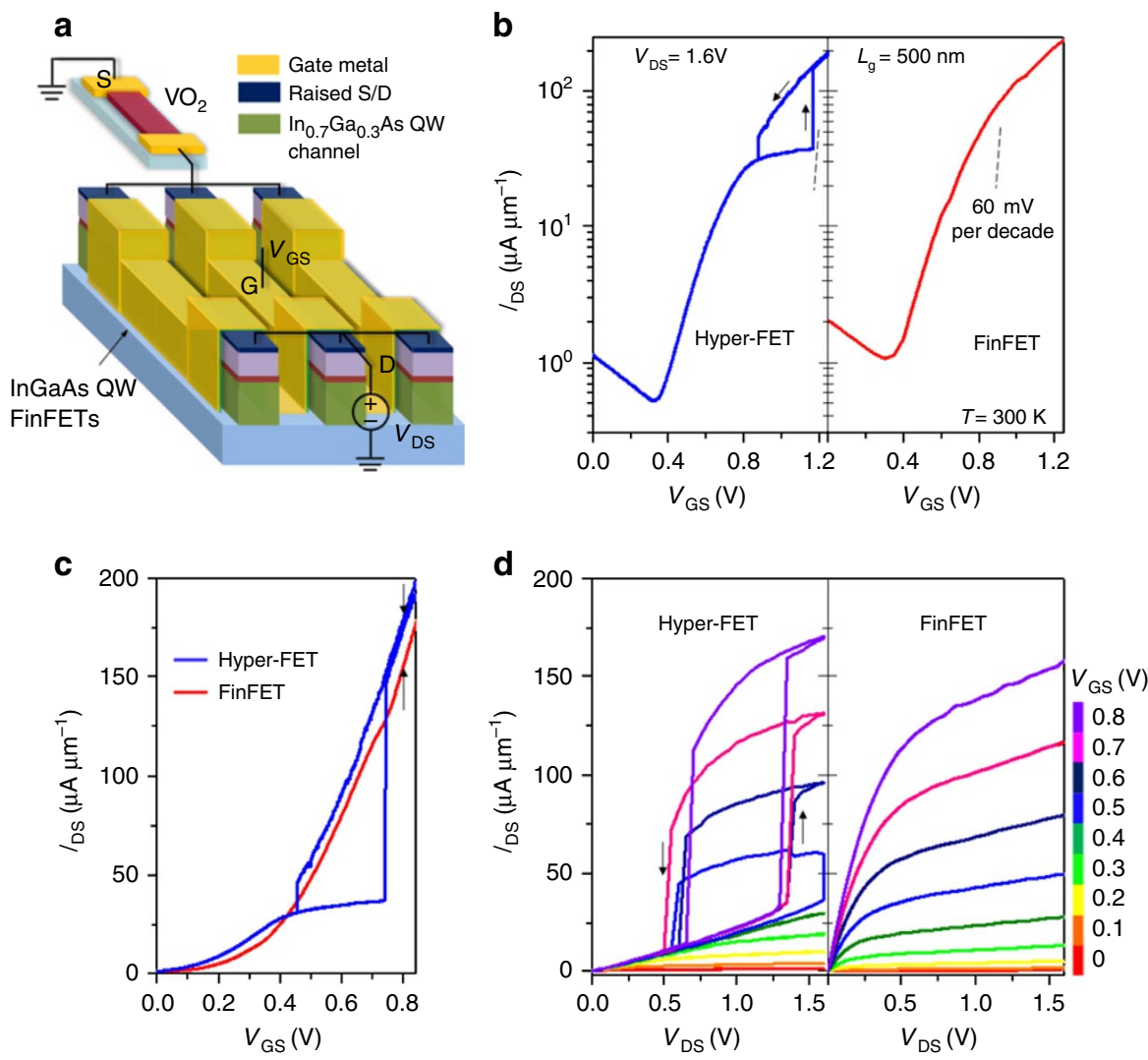

Figure 3 | Experimental demonstration a low-voltage hyper-FET using next generation FinFET technology beyond Si. (a) Schematic of the n-hyper-FET consisting of a series combination of a scaled $\mathrm{VO}_{2}\left(L_{\mathrm{VO}_{2}}=200 \mathrm{~nm}\right)$ and a multi-channel ( $=3$ fins $) \ln _{0.7} \mathrm{Ga}_{0.3} \mathrm{As}$ quantum-well FinFET $\left(L_{\mathrm{g}}=500 \mathrm{~nm}\right.$ ). (b) Transfer characteristics $\left(I_{\mathrm{DS}}-V_{\mathrm{GS}}\right.$ ) of the hyper-FET and the stand-alone FinFET. (c) The positive feedback provided by the $\mathrm{VO}_{2}$ enables the hyper-FET to exhibit a $\sim 20 \%$ higher ON-state current ( $\left.I_{D S, O N}\right)$ compared with the stand-alone $n$-FinFET over a gate-voltage window of $0.8 \mathrm{~V}$ at matched OFF-state current. (d) Output characteristics $\left(I_{\mathrm{DS}}-V_{\mathrm{DS}}\right)$ of the n-hyper-FET and the conventional FinFET.

Equation (2) indicates that in a particular gate-voltage window, the amplified differential transconductance $\left(\beta g_{\mathrm{m}} ; \beta>1\right)$ of the hyper-FET facilitates a larger change in current compared with the stand-alone MOSFET. The $\mathrm{VO}_{2}$, therefore, sets up an internal amplifier $(\beta>1)$ in the hyper-FET, and the transconductance enhancement $\left(\beta g_{\mathrm{m}}\right)$ is directly related to the free-carrier amplification across the phase transition. We note that although the hyper-FET has reduced transconductance before and after the IMT (that is, $\mathrm{VO}_{2}$ in the stable insulating/metallic state), the abrupt free-carrier amplification across the IMT overcompensates this reduction and enables an amplified current change. To evaluate the improved performance of the hyper-FET, particularly for digital-logic applications, we match the OFF-state current $I_{\mathrm{DS}, \mathrm{OFF}}$ of the hyper-FET and the stand-alone MOSFET and analyze the increase in ON-state current $I_{\mathrm{DS}, \mathrm{ON}}$ over the operating gate-voltage window, as shown further.

Low-voltage n-type and p-type hyper-FET operation. Next, we focus on the MOSFET component of the hyper-FET. The gate-bias triggers the phase transition in $\mathrm{VO}_{2}$ by enabling the MOSFET to source the corresponding critical currents. Therefore, using a scaled transistor can enable low-voltage hyper-FET operation since the transistor can now source the same currents at low $V_{\mathrm{GS}}$ and $V_{\mathrm{DS}}$. This motivates the integration of scaled, high- $g_{\mathrm{m}}$-advanced transistor architectures like FinFETs fabricated on channel materials having mobilities higher than that of silicon to design a low-voltage hyper-FET (Figs 3 and 4).

Figure 3a illustrates a scaled hyper-FET consisting of a scaled $\mathrm{In}_{0.7} \mathrm{Ga}_{0.3} \mathrm{As}$ quantum-well multi-channel FinFET $\left(L_{\mathrm{g}}=500 \mathrm{~nm}\right)$ (see Supplementary Fig. 4 and Supplementary Note 4 for fabrication method $)$ in series with $\mathrm{VO}_{2}\left(L_{\mathrm{VO}_{2}}=200 \mathrm{~nm} ; W_{\mathrm{VO}_{2}}=1\right.$ $\mu \mathrm{m})$. Figure $3 \mathrm{~b}$ shows the transfer characteristics of the hyper-FET (and the stand-alone FinFET) exhibiting a 'gate controlled' abrupt turn-ON/turn-OFF associated with the IMT/MIT in $\mathrm{VO}_{2}$, respectively. The direct comparison of the hyper-FET with the stand-alone FinFET reveals an improved $I_{\mathrm{DS}, \mathrm{ON}} / I_{\mathrm{DS}, \mathrm{OFF}}$ ratio over a $V_{\mathrm{GS}}$ range of $0.8 \mathrm{~V}$, and thus a $\sim 20 \%$ enhancement in $I_{\mathrm{DS}, \mathrm{ON}}$ at matched $I_{\mathrm{DS}, \mathrm{OFF}}$ (Fig. 3c). The corresponding output characteristics of the hyper-FET and its constituent FinFET, shown in Fig. 3d, also reflect the $I_{\mathrm{DS}}$ enhancement.

We also demonstrate a p-type hyper-FET since complementary operation, similar to the complementary metal-oxidesemiconductor (CMOS) logic family, is imperative for low standby-power digital applications. Two-terminal $\mathrm{VO}_{2}$ devices exhibit reversible switching in both positive and negative voltage polarities (Supplementary Fig. 1) which allows for electrical integration with a p-channel FinFET to enable p-type hyper-FET operation. Figure 4a shows the schematic of a p-hyper-FET constructed using a p-channel Ge quantum-well multi-channel FinFET (see Supplementary Fig. 4 and Supplementary Note 4 for fabrication method) in series with $\mathrm{VO}_{2}\left(L_{\mathrm{VO}_{2}}=200 \mathrm{~nm} ; W_{\mathrm{VO}_{2}}=1 \mu \mathrm{m}\right)$. Figure $4 \mathrm{~b}, \mathrm{~d}$ shows the transfer characteristics and the corresponding output characteristics of the p-hyper-FET and its constituent FinFET, respectively. The p-hyper-FET also exhibits an enhanced $I_{\mathrm{SD}, \mathrm{ON}} / I_{\mathrm{SD}, \mathrm{OFF}}$ ratio over a $V_{\mathrm{GS}}$ range of $-0.5 \mathrm{~V}$, and thus a $\sim 60 \%$ enhancement in $I_{\mathrm{SD}, \mathrm{ON}}$ at matched $I_{\mathrm{SD}, \mathrm{OFF}}$ (Fig. 4c). 
a

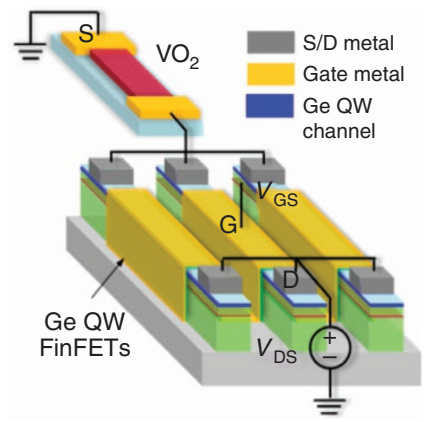

C

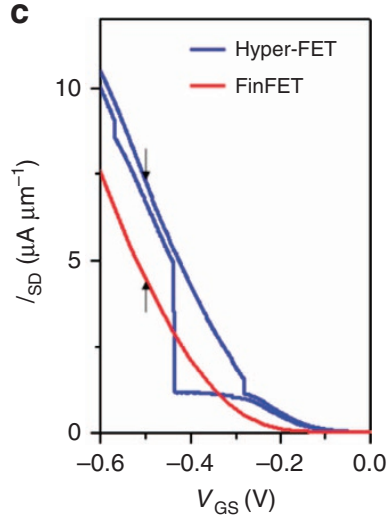

b

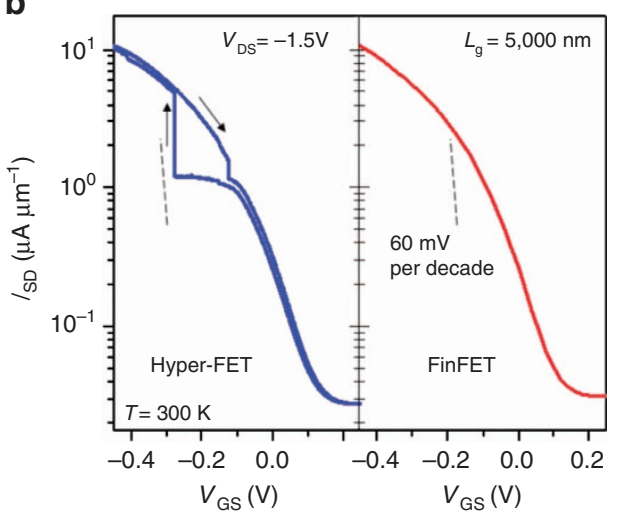

d

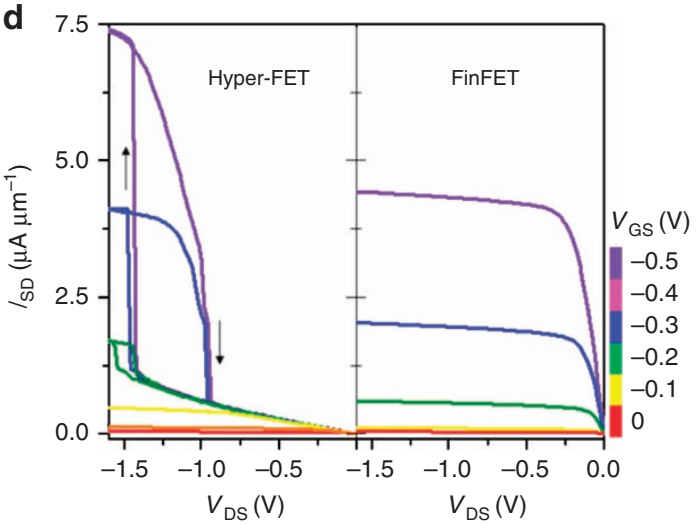

Figure 4 | Experimental demonstration of a low-voltage p-type hyper-FET. (a) Schematic of the p-hyper-FET consisting of a series combination of scaled $\mathrm{VO}_{2}\left(L_{\mathrm{VO}_{2}}=200 \mathrm{~nm}\right)$ and multi-channel ( $=200$ fins) p-type Ge quantum-well FinFET $\left(L_{\mathrm{g}}=5,000 \mathrm{~nm}\right)$. (b) Transfer characteristics $\left(I_{\mathrm{SD}}-V_{\mathrm{GS}}\right)$ of the hyper-FET and the FinFET (stand-alone). (c) The p-hyper-FET shows a $\sim 60 \%$ higher ON-state current $\left(I_{S D, O N}\right)$ in comparison to the stand-alone FinFET over a gate-voltage window of $-0.5 \mathrm{~V}$ at matched OFF-state current. (d) Output characteristics $\left(I_{\mathrm{SD}}-V_{\mathrm{DS}}\right)$ of the p-hyper-FET and the conventional FinFET.

\section{Discussion}

The hyper-FET is a device concept that harnesses the phase transition in the IMT material, $\mathrm{VO}_{2}$, to enable room temperature, steep-slope, $\mathrm{n}$-type and p-type transistor operation with enhanced performance. These experimental results motivate the realization of a scaled, monolithic hyper-FET design entailing heterointegration of the IMT material with the conventional $\mathrm{FET}^{44-48}$. Such an integrated device would have to include careful design considerations for minimizing the device 'foot-print ${ }^{\text {'4 }}$, reducing potential self-heating effects as well as ensuring low-contact resistance of both the conventional MOSFET, which can adversely affect its ON-state current (and therefore that of the hyper-FET), and that of the $\mathrm{VO}_{2}$, which may possibly affect the magnitude of abrupt current change across the IMT. Further, scaling and optimizing the $\mathrm{VO}_{2}$ and the MOSFET properties to enable a scaled hyper-FET device with low OFFstate leakage current relevant to low-power circuit applications ${ }^{49}$, and reduced hysteresis with a complete rail-to-rail swing in a complementary configuration (some of the design considerations are discussed in Supplementary Note 5 and Supplementary Fig. 5) will be key factors in realizing a hyper-FET-based hardware platform that can augment current state-of-the-art technology 49 .

The hyper-FET, demonstrated here, is a manifestation of a design methodology that consolidates the unique properties of phase transition materials like abrupt and reversible resistivity switching, arising from collective carrier dynamics and usually inaccessible in a conventional semiconductor, with the robust field-induced switching dynamics of a conventional MOSFET. Our approach harnesses the abrupt IMT in $\mathrm{VO}_{2}$ in the muchdesired three-terminal transistor configuration, circumventing the need for direct electric field-induced phase transition. Furthermore, the generality of the hyper-FET design also facilitates this transistor architecture to be extended to other insulator-metal transition systems, ${ }^{50-52}$ thus opening the doors to using electronic phase transition materials in digital applications.

\section{References}

1. Ionescu, A. M. \& Riel, H. Tunnel field-effect transistors as energy-efficient electronic switches. Nature 479, 329-337 (2011).

2. Zhirnov, V. V. \& Cavin, R. K. Nanoelectronics: negative capacitance to the rescue? Nat. Nanotechnol. 3, 77-78 (2008).

3. Markov, I. L. Limits on fundamental limits to computation. Nature 512, 147-154 (2014).

4. Cavin, R. K., Lugli, P. \& Zhirnov, V. V. Science and engineering beyond Moore's law. Proc. IEEE 100, 1720-1749 (2012).

5. Mannhart, J. \& Haensch, W. Put the pedal to the metal. Nature 487, 436-437 (2012).

6. Reich, E. S. Metal oxide chips show promise. Nature 495, 17 (2013).

7. Zhou, Y. \& Ramanathan, S. Correlated electron materials and field effect transistors for logic: a review. Crit. Rev. Solid State Mater. Sci. 38, 286-317 (2013).

8. Inoue, I. H. \& Rozenberg, M. J. Taming the Mott transition for a novel Mott transistor. Adv. Funct. Mater. 18, 2289-2292 (2008).

9. Newns, D. M. et al. Mott transition field effect transistor. Appl. Phys. Lett. 73, 780-782 (1998).

10. Bernstein, K., Cavin, R. K., Porod, W., Seabaugh, A. C. \& Welser, J. Device and architectures outlook for beyond CMOS switches. Proc. IEEE 98, 2169-2184 (2010).

11. Inoue, I. H. Electrostatic carrier doping to perovskite transition-metal oxides. Semicond. Sci. Technol. 20, S112-S120 (2005).

12. Ahn, C. H., Triscone, J.-M. \& Mannhart, J. Electric field effect in correlated oxide systems. Nature 424, 1015-1018 (2003).

13. Chakhalian, J., Millis, A. J. \& Rondinelli, J. Whither the oxide interface. Nat. Mater. 11, 92-94 (2012). 
14. Imada, M., Fujimori, A. \& Tokura, Y. Metal-insulator transitions. Rev. Mod. Phys. 70, 1039-1263 (1998).

15. Morin, F. J. Oxides which show a metal-to-insulator transition at the Neel temperature. Phys. Rev. Lett. 3, 34-36 (1959).

16. Berglund, C. N. \& Guggenheim, H. J. Electronic properties of $\mathrm{VO}_{2}$ near the semiconductor-metal transition. Phys. Rev. 185, 1022-1033 (1969).

17. Wentzcovitch, R. M., Schulz, W. W. \& Allen, P. B. $\mathrm{VO}_{2}$ : Peierls or Mott-Hubbard? A view from band theory. Phys. Rev. Lett. 72, 3389-3392 (1994).

18. Rice, T. M., Launois, H. \& Pouget, J. P. Comment on ' $\mathrm{VO}_{2}$ : Peierls or Mott-Hubbard? A view from band theory'. Phys. Rev. Lett. 73, 3042 (1994).

19. Qazilbash, M. M. et al. Mott transition in $\mathrm{VO}_{2}$ revealed by infrared spectroscopy and nano-imaging. Science 318, 1750-1753 (2007).

20. Zhang, S., Chou, J. Y. \& Lauhon, L. J. Direct correlation of structural domain formation with the metal insulator transition in a $\mathrm{VO}_{2}$ nanobeam. Nano Lett. 9, 4527-4532 (2009)

21. Cao, J. et al. Strain engineering and one-dimensional organization of metal-insulator domains in single-crystal vanadium dioxide beams. Nat. Nanotechnol. 4, 732-737 (2009).

22. Park, J. H. et al. Measurement of a solid-state triple point at the metal-insulator transition in $\mathrm{VO}_{2}$. Nature 500, 431-434 (2013).

23. Kim, H.-T. et al. Mechanism and observation of Mott transition in $\mathrm{VO}_{2}$-based two- and three-terminal devices. New J. Phys. 6, 052 (2004).

24. Kim, B.-J. et al. Micrometer X-ray diffraction study of $\mathrm{VO}_{2}$ films: separation between metal-insulator transition and structural phase transition. Phys. Rev. B 77, 235401 (2008).

25. Cao, J. et al. Constant threshold resistivity in the metal-insulator transition of $\mathrm{VO}_{2}$. Phys. Rev. B 82, 241101 (2010).

26. Zimmers, A. et al. Role of thermal heating on the voltage induced insulator-metal transition in $\mathrm{VO}_{2}$. Phys. Rev. Lett. 110, 056601 (2013).

27. Freeman, E. et al. Nanoscale structural evolution of electrically driven insulator to metal transition in vanadium dioxide. Appl. Phys. Lett. 103, 263109 (2013).

28. Stefanovich, G., Pergament, A. \& Stefanovich, D. Electrical switching and Mott transition in $\mathrm{VO}_{2}$. J. Phys. Condens. Matter 12, 8837-8845 (2000).

29. Ruzmetov, D., Heiman, D., Claflin, B., Narayanamurti, V. \& Ramanathan, S. Hall carrier density and magnetoresistance measurements in thin-film vanadium dioxide across the metal-insulator transition. Phys. Rev. B 79, 153107 (2009).

30. Ladd, L. A. \& Paul, W. Optical and transport properties of high quality crystals of $\mathrm{V}_{2} \mathrm{O}_{4}$ near the metallic transition temperature. Solid State Commun. 7, 425-428 (1969).

31. Ruzmetov, D., Gopalakrishnan, G., Ko, C., Narayanamurti, V. \& Ramanathan, $\mathrm{S}$. Three-terminal field effect devices utilizing thin film vanadium oxide as the channel layer. J. Appl. Phys. 107, 114516 (2010).

32. Boriskov, P. P., Velichko, A. A., Pergament, A. L., Stefanovich, G. B. \& Stefanovich, D. G. The effect of electric field on metal-insulator phase transition in vanadium dioxide. Tech. Phys. Lett. 28, 406-408 (2002).

33. Sengupta, S. et al. Field-effect modulation of conductance in $\mathrm{VO}_{2}$ nanobeam transistors with $\mathrm{HfO}_{2}$ as the gate dielectric. Appl. Phys. Lett. 99, 062114 (2011)

34. Nakano, M. et al. Collective bulk carrier delocalization driven by electrostatic surface charge accumulation. Nature 487, 459-462 (2012).

35. Liu, K. et al. Dense electron system from gate-controlled surface metal-insulator transition. Nano Lett. 12, 6272-6277 (2012).

36. Shi, J., Ha, S. D., Zhou, Y., Schoofs, F. \& Ramanathan, S. A correlated nickelate synaptic transistor. Nat. Commun. 4, 2676 (2013).

37. Shi, J., Zhou, Y. \& Ramanathan, S. Colossal resistance switching and band gap modulation in a perovskite nickelate by electron doping. Nat. Commun. 5, 4860 (2014).

38. Hatano, T. et al. Gate control of electronic phases in a quarter-filled manganite. Sci. Rep 3, 2904 (2013).

39. Scherwitzl, R. et al. Electric-field control of the metal-insulator transition in ultrathin $\mathrm{NdNiO}_{3}$ films. Adv. Mater. 22, 5517-5520 (2010).

40. Zhou, Y. \& Ramanathan, S. Relaxation dynamics of ionic liquid- $-\mathrm{VO}_{2}$ interfaces and influence in electric double-layer transistors. J. Appl. Phys. 111, 084508 (2012)
41. Misra, R., McCarthy, M. \& Hebard, A. F. Electric field gating with ionic liquids. Appl. Phys. Lett. 90, 052905 (2007).

42. Yi, H. T., Gao, B., Xie, W., Cheong, S.-W. \& Podzorov, V. Tuning the metal-insulator crossover and magnetism in $\mathrm{SrRuO}_{3}$ by ionic gating. Sci. Rep. 4, 6604 (2014).

43. Jeong, J. et al. Suppression of metal-insulator transition in $\mathrm{VO}_{2}$ by electric field-induced oxygen vacancy formation. Science 339, 1402-1405 (2013).

44. Lee, M.-J. et al. Two series oxide resistors applicable to high speed and high density nonvolatile memory. Adv. Mater. 19, 3919-3923 (2007).

45. Chudnovskii, F. A., Odynets, L. L., Pergament, A. L. \& Stefanovich, G. B. Electroforming and switching in oxides of transition metals: the role of metal-insulator transition in the switching mechanism. J. Solid State Chem. 122, 95-99 (1996).

46. Sakai, J. et al. Pulsed laser-deposited $\mathrm{VO}_{2}$ thin films on Pt layers. J. Appl. Phys. 113, 123503 (2013).

47. Martens, K. et al. The $\mathrm{VO}_{2}$ interface, the metal-insulator transition tunnel junction, and the metal-insulator transition switch On-Off resistance. J. Appl. Phys. 112, 124501 (2012).

48. Cheng, C. et al. Self-assembly and horizontal orientation growth of $\mathrm{VO}_{2}$ nanowires. Sci. Rep. 4, 5456 (2014).

49. Narasimha, S. et al. in IEEE International Electron Devices Meeting, 3.3.1-3.3.4 (San Francisco, 2012).

50. Brockman, J. S. et al. Subnanosecond incubation times for electric-field-induced metallization of a correlated electron oxide. Nat. Nanotechnol. 9, 453-458 (2014).

51. Pickett, M. D. \& Williams, R. S. Sub-100 fJ and sub-nanosecond thermally driven threshold switching in niobium oxide crosspoint nanodevices. Nanotechnology 23, 215202 (2012).

52. Asamitsu, A., Tomioka, Y., Kuwahara, H. \& Tokura, Y. Current switching of resistive states in magnetoresistive manganites. Nature 388, 50-52 (1997).

\section{Acknowledgements}

This work was primarily supported by the Office of Naval Research through award N00014-11-1-0665 and Intel Corporation through a customized Semiconductor Research Corporation project at the Pennsylvania State University. This work was also supported, in part, by the Center for Low Energy Systems Technology (LEAST), one of six centres of STARnet, a Semiconductor Research Corporation program sponsored by MARCO and DARPA.

\section{Author contributions}

N.S. performed the experiments. N.S. and S.D. analysed the data. A.V.T. fabricated the $\mathrm{VO}_{2}$ devices and the n-InGaAs quantum-well FinFETs. A.Ag. fabricated the p-Ge quantum-well FinFETs. H.P. grew the $\mathrm{VO}_{2}$ films. A.Az. helped with the data analysis. D.G.S., S.K.G., R.E-H. and S.D. supervised the study. N.S., S.K.G., R.E-H. and S.D. wrote the manuscript. All authors discussed the results and commented on the manuscript. S.D. directed the overall project.

\section{Additional information}

Supplementary Information accompanies this paper at http://www.nature.com/ naturecommunications

Competing financial interests: The authors declare no competing financial interests

Reprints and permission information is available online at http://npg.nature.com/ reprintsandpermissions/

How to cite this article: Shukla, N. et al. A steep-slope transistor based on abrupt electronic phase transition. Nat. Commun. 6:7812 doi: 10.1038/ncomms8812 (2015)

This work is licensed under a Creative Commons Attribution 4.0 International License. The images or other third party material in this article are included in the article's Creative Commons license, unless indicated otherwise in the credit line; if the material is not included under the Creative Commons license, users will need to obtain permission from the license holder to reproduce the material. To view a copy of this license, visit http://creativecommons.org/licenses/by/4.0/ 